\title{
Economic impact of the Swiss Diagnosis-Related Group system on acute neurorehabilitation
}

\author{
Loric Berney*1, Fabio Agri ${ }^{2}$, Jean-Michel Pignat ${ }^{3}$, Jean-Blaise Wasserfallen ${ }^{4}$, Karin Diserens ${ }^{1}$ \\ ${ }^{1}$ Acute Neurorehabilitation Unit, Department of Clinical Neurosciences, Lausanne University Hospital (CHUV), Switzerland \\ ${ }^{2}$ Department of Administration and Finance, Lausanne University Hospital (CHUV), Switzerland \\ ${ }^{3}$ Service of Neuropsychology and Neurorehabilitation, Department of Clinical Neurosciences Lausanne University Hospital \\ (CHUV), Switzerland \\ ${ }^{4}$ Medical directorate, Lausanne University Hospital (CHUV), Switzerland
}

Received: February 26, 2021

DOI: $10.5430 /$ jha.v10n4p10
Accepted: June 2, 2021

Online Published: June 20, 2021

\begin{abstract}
Objective: To assess the economic impact of introducing the Swiss Diagnosis-Related Group (DRG)-financing system on the Acute Neurorehabilitation Unit (ANRU) of a University hospital in 2012 and to discuss the implications in 2020.

Methods: A retrospective study using monocentric patient cohort and collecting anonymized data of consecutive patients admitted to the ANRU in 2012 and 2013. The characteristics, DRG A43Z, costs and revenues were retrieved from the hospital accounting system and allowed a comparison between the 2012 and 2013 groups of patients.

Results: Forty-seven patients were included over the assessment period. In 2012, of the 23 patients admitted, 20 were coded A43Z, while in 2013, out of the 24 admissions, only eight had that specific code $(p<.01)$. The average length of stay (LOS) increased from 45.5 days in 2012 to 49.5 days in 2013. Similarly, the average cost per patient increased by Swiss Franc (CHF) 19,994 over the two years, from CHF 183,634 in 2012 to CHF 194,629 in 2013. Finally, the average reimbursement per patient diminished by CHF 11,392, from CHF 193,153 in 2012 to CHF 181,760 in 2013.

Conclusions: The negative impact on the cost-revenue balance is linked to both the increased cost of a longer stay and the decreased revenue due to less patients being coded A43Z. This study highlights the difficulties to justify funding of the complex care needed and to properly reflect patient burden in medico-administrative documents. Certainly, there is a need for a concerted effort to identify the services and resources needed within the DRG-system to guarantee the optimal management of acute neurorehabilitation.
\end{abstract}

Key Words: Diagnosis-related group, Acute neurorehabilitation, Cost-revenue balance, Economic impact

\section{INTRODUCTION}

On January 1st 2012, all hospitals in Switzerland adopted the new Diagnosis-Related Group (DRG) reimbursement system for acute inpatient hospital services. The SwissDRG is based on diagnostic (International Classification of Diseases, ICD-10) and intervention (Swiss Classification of Surgical
Interventions, $\mathrm{CHOP}) \operatorname{codes}^{[1]}$ and regulates the remuneration of inpatient hospital services under a fixed rate-per-case payment schedule.

Classification into a DRG is assigned by a pooling software application called "DRG grouper" that considers diagnostics, intervention codes and associated data (gender, age, source

\footnotetext{
*Correspondence: Loric Berney; Email: loric.berney@chuv.ch; Address: Acute Neurorehabilitation Unit, Department of Clinical Neurosciences (BH13-545), Lausanne University Hospital (CHUV), Rue du Bugnon 46, CH-1011 LAUSANNE, Switzerland.
} 
and destination, pre- and post-hospitalization, nationality, admission mode, for example). The cost-weight $(\mathrm{CW})$ is calculated as the ratio of the average cost of all cases in the same DRG to the average cost of all cases across all DRGs. The final remuneration is calculated by multiplying the $\mathrm{CW}$ of the DRG with the hospital-specific base rate (BR). The $\mathrm{CW}$ is recalculated annually based on the costs incurred by the DRG in Swiss hospitals in the previous two years and the $\mathrm{BR}$ is set annually by the cantons if no agreement is found by the tariff partners (insurers and care providers).

For acute neurorehabilitation, a procedure shown to increase the survival rate of patients with severe central or peripheral neurologic damage caused by accident or disease, it is possible to bill for a specific DRG code, called A43Z. This occurs when ICD-10 diagnostic codes G83.80 (locked-in syndrome) or G93.80 (apallic syndrome) are combined with the intervention code CHOP 93.89.1 (early neurological or neurosurgical rehabilitation). ${ }^{[2,3]}$ This well-defined DRG code (A43Z) designates an early rehabilitation program for coma recovery and locked-in syndrome. The $\mathrm{CW}$ generated by associating one of the above two ICD-10 diagnostic codes with the intervention code CHOP 93.89.1 was negotiated for the years 2012 and 2013 as equivalent to 20.223 points, or approximately CHF 200000 per admitted patient. However, combining intervention code CHOP 93.89.1 with other ICD10 diagnostic codes, such as I63.4 (Cerebral infarction due to embolism of cerebral arteries) leads to other DRG codes, including B42A, B42B, B11Z and B43Z, which depend on the patient's length of stay (LOS). All the latter DRG codes have lower associated CWs than the CW of DRG A43Z (see Table 1).

In 2001, the Lausanne area hospital established a clinical pathway for neurorehabilitation followed in 2009 by the opening of an Acute Neurorehabilitation Unit (ANRU), both managed by a multidisciplinary team composed of physi- cians, physical therapists, occupational therapists, speech specialists, neuropsychologists and specialized nurses and headed by a coordinator. ${ }^{[4]}$ The multidisciplinary team coordinates the care of selected patients transferred from the intensive care unit to the ANRU. The positive impact of this team on patient outcome has been indisputably demonstrated on a specific patient population; patients with tracheostomies ${ }^{[5]}$ On January 1st, 2012, two specific intermediate acute-care beds were added to the ANRU. This unit is the only one of its kind existing in an acute-care hospital setting in Switzerland. Hence, no benchmark was available to adapt the CW of DRG A43Z within the adopted SwissDRG system.

In other countries, the introduction of DRGs has had a significant impact on hospitals, notably a considerable decrease in the LOS in the acute phase. ${ }^{[6]}$ Here, we aim to assess the economic impact of introducing the SwissDRG financing system on the ANRU in the Lausanne area hospital.

\section{MeThods}

\subsection{Data}

Anonymized data (intra-hospital numbers) of all consecutive patients admitted to the ANRU in 2012 and in 2013 were included in the analysis. Length of hospital stay with the corresponding LOS in the different hospital units was obtained from the hospital information system. As patient data were not used in the study, approval from the institutional ethics review board was not required. ICD-10 diagnostic and CHOP intervention codes were used to classify into the corresponding DRG. Further, for each patient's stay, direct medical costs were retrieved from the hospital accounting system using micro-costing approach for precise estimation. ${ }^{[7]}$

Revenues were obtained from the corresponding DRG according to the 2012 and 2013 annual-base rates and the balance between cost and revenue was computed for each patient.

Table 1. Referenced DRGs' characteristics

\begin{tabular}{|c|c|c|c|c|}
\hline \multirow{2}{*}{ DRG } & \multirow{2}{*}{ Designation } & \multirow{2}{*}{ Cost-weight } & \multirow{2}{*}{$\begin{array}{l}\text { LOS (day, } \\
\text { average) }\end{array}$} & \multirow{2}{*}{$\begin{array}{l}\text { Payment (CHF) } \\
\text { (BR) CHF } 10600\end{array}$} \\
\hline & & & & \\
\hline A43Z & Early rehabilitation for vigil coma and locked-in syndrome & 20.223 & 53.7 & 214,364 \\
\hline B11Z & $\begin{array}{l}\text { Rehabilitation with craniotomy, major spinal surgery, special operative procedure or } \\
\text { expensive operation on the nervous system with artificial ventilation }>95 \text { hours }\end{array}$ & 8.519 & 36.2 & 90,301 \\
\hline B42A & $\begin{array}{l}\text { Early rehabilitation for diseases and disorders of the nervous system up to } 27 \text { days, with } \\
\text { complex neurological treatment of acute stroke }\end{array}$ & 3.65 & 25.9 & 38,690 \\
\hline B42B & $\begin{array}{l}\text { Early rehabilitation for diseases and disorders of the nervous system up to } 27 \text { days, without } \\
\text { complex neurological treatment of acute stroke }\end{array}$ & 2.877 & 21.2 & 30,422 \\
\hline B43Z & Early rehabilitation of diseases and disorders of the nervous system, over 27 days & 4 & 44.3 & 42,400 \\
\hline
\end{tabular}

Note . Payment $=$ Base rate $(\mathrm{BR}) \times$ cost-weight $(\mathrm{CW})$ 


\subsection{Statistics}

Following the cost variables, including the DRG, the LOS in the different hospital units, unit costs, hospital costs, DRG income and global profit (difference between hospital costs and DRG income) were statistically compared using Matlabß R2011a (The MathWorks, Inc., Natick, Massachusetts, USA, http: //www . mathworks. com), to test for the various cost differences between 2012 and 2013. A normality (Lilliefors) test of the groups for all cost variables was performed to guide the selection of an appropriate inferential procedure. As the null hypothesis of normality was rejected for several cost variables (for the DRG, total LOS, hospital costs and global profit variables, with $p<.005$, and after multiple $p$-value corrections using the Bonferroni-Holm method), a nonparametric test, the two-sample Wilcoxon rank-sum test, was chosen to test the hypothesis of no difference between group medians.

\section{RESUltS}

\subsection{Patients and LOS}

In 2012, 23 patients were admitted to the ANRU and 20 were coded A43Z, while in 2013, of the 24 patients admitted, only 8 were coded A43Z showing a statistically significant difference between the two years (see Table 2).

Table 2. Comparison of LOS, costs and revenues between 2012 and 2013

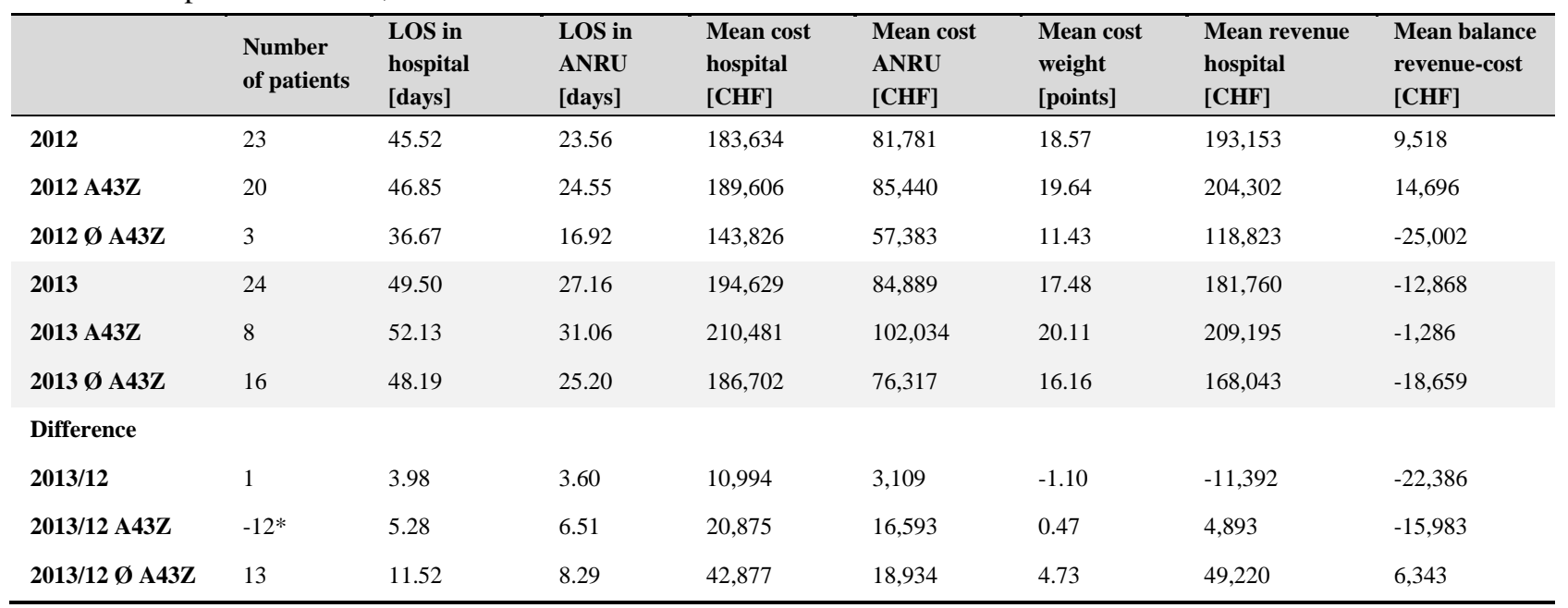

Note. $*=p<.01 ;$ LOS = length of stay; ANRU = Acute Neurorehabilitation Unit

The average hospital LOS increased from 45.5 days to 49.5 days from 2012 to 2013 and the specific ANRU LOS increased from 23.5 days in 2012 (24.5 days for A43Z patients) to 27.2 days in 2013 (31.1 days for A43Z patients), as shown in Table 2.

\subsection{Costs and revenues}

In 2012, the average cost per patient was CHF 183 634, of which the ANRU accounted for CHF 81781. In 2013, these figures were CHF 194629 and CHF 84889, respectively. The corresponding values for the specific DRG code A43Z costs are displayed in Table 2. The average hospital cost per patient increased by CHF 10994 between 2012 and 2013 On the other hand, the corresponding average revenue per patient decreased from CHF 193153 in 2012 to CHF 181 760 in 2013 (a difference of CHF 11393). Moreover, the corresponding revenue from the DRG code A43Z remained stable between the two years, increasing slightly by $\mathrm{CHF}$ 5000. Further, no statistically significant differences were observed.

12

\section{Discussion}

\subsection{Impact of analysis of the SwissDRG reimbursement system for ANRU patients}

The impact of the SwissDRG reimbursement system on the acute neurorehabilitation unit between 2012 and 2013 showed some interesting features. The average total LOS increased by 3.6 days, while that in the acute neurorehabilitation unit increased by 6.5 days. This was ascribed to the requirement of the CHOP intervention code 93.89.11 for a minimum LOS of 14 days for coding DRG A43Z in 2013, while there was no defined minimal LOS in 2012. A major difficulty with the DRG system, probably related to the introduction of a new arrangement, is that coding rules change every year. As a result, the inclusion or not of patients eligible for A43Z coding also changes, which may induce an increase in LOS. Considering the positive effect of the system on hospitals in other countries with concomitant decrease in LOS, perhaps the system is not optimal for this class of patient. Studies carried out in Switzerland and 
Germany do not make it possible to establish a relationship between the introduction of the DRG system and a shorter LOS, particularly in tertiary referral hospitals. ${ }^{[8]}$

The number of patients coded DRG A43Z dropped markedly in 2013 compared to 2012. This translated into an important decrease in the average $\mathrm{CW}$ for all patients in 2013, while the average $\mathrm{CW}$ for the DRG code A43Z remained stable $(+0.47)$. Four reasons explain this difference. First, the admission criteria to the ANRU were broadened; implying that patients with ICD-10 diagnostic codes other than G83.80 or G93.80 were admitted and who then were not assigned DRG code A43Z. Second, five patients were mistakenly not coded as DRG A43Z. Third, insurance companies opposed some bills with DRG code A43Z. Lastly, some patients with ICD-10 diagnostic codes G83.80 or G93.80 were not coded DRG A43Z because their LOS in the ANRU was shorter than the minimal requirement of 14 days.

In this situation, three important measures are necessary. First, a defined clinical diagnosis of the minimally conscious state should be recognized to allow for coding DRG A43Z. Second, all coding errors must be prevented. Third, given the small number of beds available, better patient selection should be applied, targeting patients who satisfy the criteria for DRG code A43Z. These measures are especially important because the acute neurorehabilitation unit of the Lausanne university hospital is the only specialized acute neurorehabilitation framework currently in existence in Switzerland. Consequently, at present we cannot establish a benchmark to guide the negotiation of DRG code A43Z CW. As the CW decreased in 2014 by 2.59 points, (meaning a decrease in revenue of about CHF 25000 per patient) and further decreased by 3.81 points in 2015 (approximately CHF 40000 per patient), the expected loss in revenue amounted to CHF 65000 per patient over two years. This potentially threatens the survival of this type of patient treatment. Therefore, support for a SwissDRG decision group of acute neurorehabilitation experts, as well as for those working in neurorehabilitation around the world seems warranted. Similarly, analogous units created in other hospitals could provide useful standards for future $\mathrm{CW}$ negotiations.

The findings of this study led to closer collaboration between the rehabilitation unit and the hospital coding center. Indeed, the continuous challenge to justify the code $\mathrm{A} 43 \mathrm{Z}$ has grown in recent years, needing even more resources in 2020 to properly reflect the burden of the patient treated in the ANRU and the care delivered.

\subsection{Limitations}

Limitations of the study include its retrospective nature. In addition, sample size was limited by the scarce number of beds in this unit. The analysis, based on the anonymized data obtained from the hospital information system did not consider the variables related to the admitted patients (e.g., age and diagnoses) that could influence the results. Further, the analysis was carried out in a single institution and even though it may be possible to extend it to other Swiss university hospitals, it will probably not be widely generalizable.

\section{CONClusions AND IMPlications}

The economic impact of the SwissDRG system on specific activities like acute neurorehabilitation, is likely to be important as the DRG rules change annually, which may have unattended consequences on revenue and eventually on the cost-revenue balance of treating the concerned patients. This kind of assessment should be systematically undertaken in each hospital, notably for improving their strategic activities.

In addition, the work carried out within the acute neurorehabilitation unit is strongly supported by the World Federation of NeuroRehabilitation (WFNR) that identified Switzerland as an international pilot project in a medical field that needs to find its place in the acute environment in order to monitor progress in surgical and medical care. In this respect, it is essential to assess and monitor the impact of SwissDRG on this acute neurorehabilitation unit every year. Funding to cover the costs will ensure the sustainability of efforts to service patients requiring acute neurorehabilitation and allow other university hospitals to set up similar units throughout the country. A financial valorization of neurorehabilitation services during the acute phase, as for example the financing system envisaged for post-acute rehabilitation centers in 2022 , is essential to fortify staff and improve the quality of care. Validating with other hospitals would allow increased efficiency.

Further analysis of the significant proportion of patients consuming neurorehabilitation services in other acute departments of area hospitals, such as neurology or neurosurgery is necessary. Compared to other expensive treatments, particularly in the context of oncological treatments for example, the cost of acute neurorehabilitation that helps recover patients from coma and enable some to return home with sufficient autonomy to live for many more years, seems ultimately quite modest for the long-term benefit. Approximately 900 patients likely to benefit from neurorehabilitation are monitored annually in the ANRU. Some of them undergo intensive acute neurorehabilitation at a rate of 15 treatments per week, each lasting at least 30 minutes (complex care corresponding to another CHOP code). However, the limitation of human resources prevents offering such advantages to all patients in need. Indeed, with further resources, the interdisciplinary team could be extended, allowing "complex care" services to 
be broadened to all patients requiring them and consequently, Pinget, Pierre Phedon Tahintzi, Jane Johr, Caroline Attwell, guaranteeing optimal management of acute neurorehabilita- Stefan Studer, Richard Frackowiak, and all of the ANRU tion. In this sense, the future identification of services and resources needed is of vital importance. team for their support in this project. We would also like to thank Melanie Price Hirt for English-language editing.

\section{ACKNOWLEDGEMENTS}

The authors would like to thank Guy Moser, Christophe

\section{CONFLiCTS OF INTEREST Disclosure}

The authors declare they have no conflicts of interest.

\section{REFERENCES}

[1] Wasserfallen JB, Suffered J. Financial impact of introducing the SwissDRG reimbursement system on potentially avoidable readmissions at a university hospital. Swiss Med Wkly. 2015; 145: w14097. https://doi.org/10.4414/smw. 2015.14097

[2] Cruz-Flores S, Berge E, Whittle IR. Surgical decompression for cerebral oedema in acute ischaemic stroke. Cochrane Database Syst Rev. 2012; 18(1): CD003435. https://doi.org/10.1002/14651858 .CD003435.pub2

[3] Lu X, Huang B, Zheng J, et al. Decompressive craniectomy for the treatment of malignant infarction of the middle cerebral artery. Sci Rep. 2014; 17(4): 7070.

[4] Berney L, Wasserfallen JB, Schweizer V, et al. Early neurorehabilitation in an acute university hospital: From dream to reality. Rev Méd Suisse. 2014; 7(293): 952-956.
[5] Berney L, Wasserfallen JB, Grant K, et al. Acute neurorehabilitation: Does a neurosensory and coordinated interdisciplinary programme reduce tracheostomy weaning time and weaning failure? NeuroRehabilitation. 2014; 34(4): 809-817. https://doi .org/10.3233/NR E-141081

[6] Von Eiff W, Schüring S, Greitemann B, et al. REDIA-Impacts of DRG introduction in the acute sector on medical rehabilitation. Rehabilitation. 2011; 50(4): 214-221. https://doi.org/10.1055/ s-0031- 1275720

[7] Xu X, Grossetta Nardini HK, Prah Ruger J. Micro-costing studies in the health and medical literature: protocol for a systematic review. Syst Rev. 2014; 3: 47. https://doi.org/10.1186/2046-405 3-3-47

[8] Chok L, Bachli EB, Steiger P, et al. Effect of diagnosis-related group implementation on the intensive care unit of a Swiss tertiary hospital: a cohort study. BMC Health Serv. Res. 2018; 18(1): 84. https://doi.org/10.1186/s12913-018-2869-4 